\title{
Modified Freeman Model: A Stability Analysis and Application to Pattern Recognition
}

\author{
Mustafa C. Ozturk, Dongming Xu and José C. Principe \\ Computational NeuroEngineering Laboratory \\ Department of Electrical and Computer Engineering \\ University of Florida \\ Gainesville, FL 32611 USA \\ E-mail: $\{$ can, dmxu, principe $\}$ cnel.ufl.edu
}

\begin{abstract}
The biologically realistic Freeman model of the olfactory cortex has been used to solve some engineering problems. However, due to the nature of the nonlinear function in the model, only numerical computer simulations can help explore the behavior of the system for different sets of control parameters. In this paper, we modify the nonlinear function with a piecewise linear model and show that this simplified model exhibits the same qualitative behavior as the original one. Moreover, for this modified model, we employ the analytical tools of nonlinear dynamics to understand the system response for different parameter values. Finally, similar to the original system, we show that the modified system can be used as an auto-associative memory.
\end{abstract}

\section{INTRODUCTION}

Understanding the information processing mechanism of the brain has been a difficult challenge for neuroscientists for long time. There have been different ideas on how brain might process and respond to the massive bombardment of information via the sensory system [1], [2], [3], [4], [5]. One of the major contributions to the area has been from Walter Freeman who proposed a dynamical system representing the response of thousands of cells, called the neural assembly, instead of the response of a single neuron [6]. The model is a hierarchical network of coupled nonlinear oscillators, where the basic building block is a second order nonlinear dynamical element followed by a nonlinear function. According to the parameter selection and the input pattern, these coupled oscillators will cooperate or compete among each other creating the basics for association, and information processing in general. Freeman, in his work, used the experimental recordings to build the model and aimed to mimic the behavior of the olfaction to understand how it works [6].

On the other hand, as engineers, we aim to use this biologically plausible model to solve difficult engineering problems. Hence, it is vital to analyze the system to design it by deciding the parameters embedded in the system and control it by modifying the input from outside of the system. However, due to the nature of the nonlinear function employed in the Freeman model, only numerical computer simulations can help explore the behavior of the system for different sets of parameters. Hence, we propose to modify the nonlinear function so that we can analyze the system with the tools of nonlinear dynamics. We will call this new system as the
Modified Freeman Model. This modified model preserves the original system behavior not necessarily trying to mimic the exact olfactory system. With the analyzable nature of the modified system, we can design and control the system parameters so that the response of the system is as desired.

In this paper, we provide the mathematical results obtained from the analysis of this modified model for the KII level of the Freeman model. We use the developed tools of nonlinear dynamics to provide a complete stability analysis for different parameter sets and input values. We also justify our mathematical development with the digital simulator whose validity we confirmed previously [7]. Finally, we show that the Modified Freeman Model can function as an auto-associative memory that can store a set of patterns during learning and retrieve the stored pattern that is most similar to the present input pattern.

\section{Overview OF THE MODEL}

The Freeman model is built from basic second order nonlinear elements in a hierarchy of levels designated by $\mathrm{K} 0, \mathrm{KI}$, KII, and KIII due to the seminal work of Katchalsky [6]. The basic building block of the model is the K0 set, which is depicted in Fig. 1.

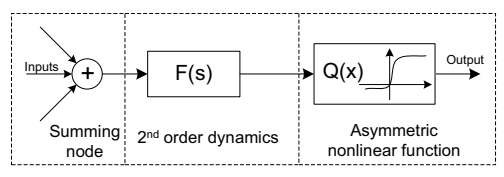

Fig. 1. Diagram of an excitatory K0 set.

Every K0 unit can accept several spatial inputs that are weighted and summed, and then convolved with a linear time invariant system defined by the second order dynamics whose transfer function, $\mathrm{H}(\mathrm{s})$, is given by

$$
H(s)=\frac{a b}{(s+a)(s+b)}
$$

where $1 / a$ and $1 / b$ are real time constants determined experimentally. The output of the linear dynamics is then shaped by the nonlinear sigmoid function (Fig. 2), which is experimentally determined to be

$$
Q(x)= \begin{cases}Q_{m} \cdot\left(1-e^{-\frac{e^{x}-1}{Q_{m}}}\right) & x>x_{0} \\ -1 & \text { else. }\end{cases}
$$


The sign of the connection strength from a K0 set will

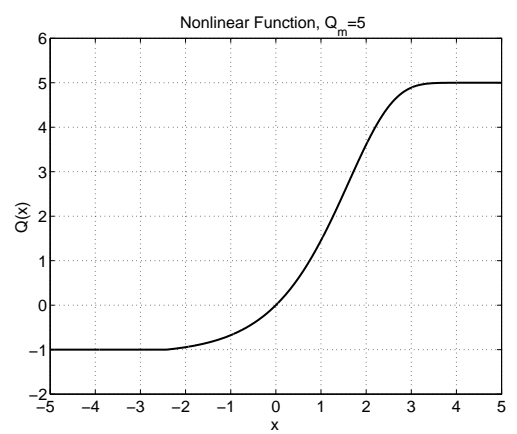

Fig. 2. Nonlinear function.

define the type of the $\mathrm{K} 0$ set (excitatory for positive weights and inhibitory for negative weights). The second level in the hierarchy is the KI set. For a detailed description of the KI set, please refer to [6].

The third level in the hierarchy, the KII set, is the most interesting and important building block of the olfactory system, since it is an oscillator controlled by the input. The response of the KII set to an impulse input is a damped oscillation whereas with a sustained input, the output oscillation is maintained as long as the input remains. The architecture of KII set is shown in Fig. 3.a), where the circles denote $\mathrm{K} 0$ sets and the sign indicates the type of connection. Fig. 3.b) shows a reduced KII set, where the two $\mathrm{K} 0$ units are denoted by M (for mitral cell) and $\mathrm{G}$ (for granular cell). In this model, the mitral cell takes the external input $I(t)$ and the coupling strengths between $\mathbf{M}$ and $\mathrm{G}$ are controlled by the two weights $K_{m g}>0$ (excitatory) and $K_{g m}<0$ (inhibitory). If the coupling weights are selected properly, the reduced KII is, similar to KII set, an oscillator controlled by the input.

A reduced KII network can be formed by fully interconnecting a number of reduced KII sets with excitatory connections between the excitatory cells (top circle in Fig. 4) and inhibitory connections between inhibitory cells (bottom circle in Fig.

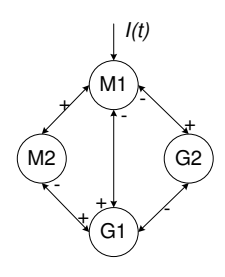

(a)

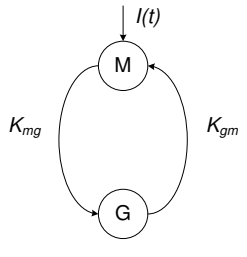

(b)
Fig. 3. Structures of KII sets. a) Full KII set. b) Reduced KII set.

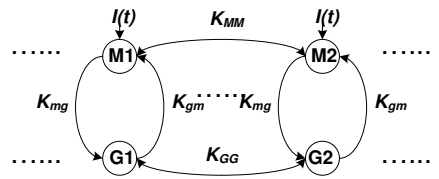

Fig. 4. Reduced KII network.
4). This interconnected structure represents a key stage of learning and memory in the olfactory system. Input patterns through $\mathrm{M}$ cells are mapped into spatially distributed outputs. Excitatory and inhibitory interconnections enable cooperative and competitive behaviors, respectively, in this network. The reduced KII network functions as an associative memory [1], [8]. The final Katchalsky level is the KIII network and represents the olfactory cortex. For a detailed description of the KIII network, we refer the reader to [1], [6].

For the purposes of this paper, we will focus on the reduced KII set. We will use the reduced KII set for the stability analysis. Hence, it is useful to state the mathematical model for the reduced KII set. The governing equations for the reduced KII set are

$$
\left\{\begin{array}{l}
\frac{1}{a b} \ddot{m}(t)+\frac{a+b}{a b} \dot{m}(t)+m(t)=K_{g m} Q(g(t))+I(t) \\
\frac{1}{a b} \ddot{g}(t)+\frac{a+b}{a b} \dot{g}(t)+g(t)=K_{m g} Q(m(t))
\end{array}\right.
$$

where $K_{m g}>0$ and $K_{g m}<0 . m$ and $g$ denote the output of the linear dynamic portions of the $\mathrm{K} 0$ units for $\mathrm{M}$ and $\mathrm{G}$, respectively [9], [10].

\section{Modified Freeman Model}

Consider the reduced KII set of the Freeman model with the governing equations stated in Eq. (3). Previously, the reduced KII set has been analyzed using the tools of nonlinear dynamics [10]. In particular, Hartman-Grobman theorem [11] has been used to analyze the stability properties of the model for different set of parameters, $K_{m g}, K_{g m}$ and input, $I(t)$. This theorem describes the condition where the phase portrait of a nonlinear dynamical system is locally qualitatively the same as that of its linearized version in a neighbourhood of equilibrium of the system. The first step to apply the Hartman-Grobman theorem is to find the equilibrium solution for a given set of parameters. For the reduced KII set, the following governing equation for the equilibrium solution is derived by equating the differential terms in Eq. (3) to zero

$$
\left\{\begin{array}{l}
m_{0}(t)=K_{g m} Q\left(g_{0}(t)\right)+I(t) \\
g_{0}(t)=K_{m g} Q\left(m_{0}(t)\right) .
\end{array}\right.
$$

However, if we use the nonlinear function given by Eq. (2), this set of equations does not lead to an explicit analytical solution for $m_{0}$ and $g_{0}$ due to the exponential terms of the nonlinearity. Instead, recursive numerical algorithms must be utilized to get a solution for the equilibrium. Hence, in order to provide a full analysis for the system, we will use a simpler piecewise linearized version of the original nonlinearity and show that this simplified model still has the same peculiar behaviour of a reduced KII set. That is, the response of the reduced KII set to an impulse input is a damped oscillation whereas with a sustained input, the output oscillation is maintained as long as the input remains. The most straightforward approach is then to use the piecewise linear curve given by

$$
Q(x)= \begin{cases}-1 & x \leq x_{1} \\ K_{s} \cdot x & x_{1}<x \leq x_{2} \\ Q_{m} & x \geq x_{2}\end{cases}
$$


where $K_{s}$ is the slope of the line and $x_{1}, x_{2}$ are the region of support of the line on the x-axis. Here, $x_{1}, x_{2}$ and $K_{s}$ has to be chosen such that the modified nonlinearity preserves the basic properties of the original nonlinearity defined by Eq. (2). Hence, we choose the nonlinearity such that it contains the point $(0,0)$ and the saturation values are -1 and +5 . For now, we do not have any other restrictions in the selection of these parameters. In order to apply the linearization, we need to find the equilibrium point for any given set of parameters and input value. Now, consider Eq. (4) that define the fixedpoint solution for the reduced KII set. It has been shown in [10] that there exists a unique solution for this set of equations with the nonlinearity given by Eq. (2). The reason is that for any possible selection of parameters, the nullclines defined by Eq. (4) intersect at a unique point due to the sigmoid shape of the nonlinearity. Similarly, we have a unique solution for the modified nonlinearity given by Eq. (4) since the nonlinearity has similar shape. If we assume that the equilibrium solution is in the linear region of the nonlinearity, $Q(x)$ can be replaced by $K_{s} \cdot x$ in Eq. (4). Then, the equilibrium solution becomes

$m_{0}=\frac{1}{1+K_{s}^{2}\left|K_{m g} K_{g m}\right|} \quad$ and $\quad g_{0}=\frac{K_{s} \cdot K_{m g} \cdot I}{1+K_{s}^{2}\left|K_{m g} K_{g m}\right|}$.

This solution is valid if and only if both $m_{0}$ and $g_{0}$ is in the linear region of the nonlinearity, i.e.

$$
x_{1}<m_{0}<x_{2} \text { and } x_{1}<g_{0}<x_{2} .
$$

Substituting in Eq. (6), we get the equivalent condition as

$$
\begin{gathered}
x_{1}\left(1+K_{s}^{2}\left|K_{m g} K_{g m}\right|\right)<I<x_{2}\left(1+K_{s}^{2}\left|K_{m g} K_{g m}\right|\right) \\
\frac{x_{1}\left(1+K_{s}^{2}\left|K_{m g} K_{g m}\right|\right)}{K_{s} K_{m g}}<I<\frac{x_{2}\left(1+K_{s}^{2}\left|K_{m g} K_{g m}\right|\right)}{K_{s} K_{m g}} .
\end{gathered}
$$

Now, how can we find the fixed point solution if any of these conditions are not satisfied? First, note that the left side of the both inequalities is less then zero since $x_{1}$ is less then zero. However, we usually do not allow the input to be less then zero, which implies that we do not have to check the left inequality. Now, without loss of generality, assume the first inequality in Eq. (8) is not satisfied, then this means that the mitral cell is saturated, i.e. $Q\left(m_{0}\right)=5$. Then using the second equation in Eq. (6), we can find $g_{0}$. Substituting $g_{0}$ in the first equation in Eq. (6), we can find $m_{0}$. So, for any given $K_{m g}, K_{g m}$ and $I$, we can find the equilibrium solution. After deciding the equilibrium point for any given setup, now we can linearize the system to check the stability of the system around the equilibrium. The system defined by Eq. (3) can be written in state-space form as

$$
\left\{\begin{array}{l}
\dot{m}_{1}=m_{2} \\
\dot{m}_{2}=-a b \cdot m_{1}-(a+b) \cdot m_{2}+a b \cdot\left(K_{g m} Q\left(g_{1}\right)+I\right) \\
\dot{g}_{1}=g_{2} \\
\dot{g}_{2}=-a b \cdot g_{1}-(a+b) \cdot g_{2}+a b \cdot K_{m g} Q\left(m_{1}\right) .
\end{array}\right.
$$

The Jacobian matrix of the system defined by Eq. (9) around an equilibrium point $\left(m_{0}, g_{0}\right)$ is [10]

$$
\begin{aligned}
& L= \\
& {\left[\begin{array}{cccc}
0 & 1 & 0 & 0 \\
-a b & -(a+b) & a b K_{g m} Q^{\prime}\left(g_{0}\right) & 0 \\
0 & 0 & 0 & 1 \\
a b K_{m g} Q^{\prime}\left(m_{0}\right) & 0 & -a b & -(a+b)
\end{array}\right]}
\end{aligned}
$$

Then, by computing the eigenvalues of Eq. (10), the condition for which there is a pair of eigenvalues with zeros real part is found as

$$
\left|K_{m g} \cdot K_{g m}\right|=\frac{1}{Q^{\prime}\left(m_{0}\right) \cdot Q^{\prime}\left(g_{0}\right)} \cdot \frac{(a+b)^{2}}{a \cdot b}
$$

This point defines the bifurcation point of the system. Similarly, $\left|K_{m g} \cdot K_{g m}\right|>\frac{1}{Q^{\prime}\left(m_{0}\right) \cdot Q^{\prime}\left(g_{0}\right)} \cdot \frac{(a+b)^{2}}{a \cdot b}$ is the condition for hyperbolic and unstable equilibrium while $\left|K_{m g} \cdot K_{g m}\right|<\frac{1}{Q^{\prime}\left(m_{0}\right) \cdot Q^{\prime}\left(g_{0}\right)} \cdot \frac{(a+b)^{2}}{a \cdot b}$ leads to stable equilibrium points [10]. For the modified nonlinearity, $Q^{\prime}\left(m_{0}\right)$ or $Q^{\prime}\left(g_{0}\right)$ is always one of the two values, zero or $m$. If it is zero (that is, the equilibrium solution is not in linear region), then, the right hand side of Eq. (11) becomes infinity and the system is stable. If the equilibrium solution is in the linear region of the nonlinearity (that is, Eq. (9) is satisfied), then the right hand side of Eq. (11) becomes a constant value, which is $\frac{(a+b)^{2}}{a b} \cdot \frac{1}{K_{s}^{2}}$. Hence, if $\left|K_{m g} \cdot K_{g m}\right|$ is less than this constant, the equilibrium is stable, else unstable. As is done in [7], [10], we will assume that if a reduced KII set is not at a bifurcation point, then its dynamical behavior is determined by the qualitative behavior at its equilibrium points. The peculiar behavior of the reduced KII set is that the output vanishes with an impulsive input, whereas a sustained input results in oscillation. Hence, we want to choose parameters $K_{m g}$ and $K_{g m}$ such that the equilibrium is unstable for a nonzero input whereas the equilibrium for a zero input should be stable. However, with this nonlinearity, a positive input can not change the stability of an equilibrium in the linear region because the slope of the operating point does not change with the input. In other words, as long as the fixed-point solution is in the linear region, only $K_{m g}$ and $K_{g m}$ determine the stability. If we set $K_{m g}$ and $K_{g m}$ so that solutions in the linear region are unstable, then an impulsive input will not cause a damped oscillation but a sustained oscillation. In order to solve this problem, we have to investigate the stability condition given by Eq. (11). We want that any equilibrium around zero is stable equilibrium, whereas any significant nonzero input results in oscillation. We can use various nonlinearities to satisfy this condition.

\section{A. Case I:}

When using a nonlinear function as shown in Eq. (5), in order to control oscillatory state by external inputs, the key is to satisfy the condition given in Eq. (11). Note that the 
bound is determined by the slope of the nonlinear function at a particular point. There are only two values available: $K_{s}$ in the linear section and 0 in the saturation regions. Thus, a negative or large positive input will possibly to make the two nullclines intersected so that Eq. (11) will never be valid with finite internal couplings. This can be done by negative external inputs. If nonnegative inputs are required, we can shift one of the nullclines as shown in Fig. 5 so that when input is small enough, the equilibrium is always stable.

The lower threshold between stable and oscillatory state is determined by

$$
I>O_{t}
$$

where theoretically $O_{t}$ could be any positive value. The external input cannot be arbitrarily large. It must guarantee that the intersection is in the linear region. By solving the equilibrium, we have the following condition on $I$

$$
I<\frac{Q_{m}}{K_{s}}+O_{t}+\min \left\{\frac{K_{m g}}{K_{g m}}, \frac{K_{g m}}{K_{m g}}\right\} \cdot \frac{Q_{m}}{K_{s}}
$$

The equilibrium is given by

$$
\left\{\begin{aligned}
m_{0} & =\frac{I+\left|K_{m g} K_{g m}\right| \cdot K_{s}^{2} \cdot O_{t}}{1+\left|K_{m g} K_{g m}\right| \cdot K_{s}^{2}} \\
g_{0} & =\frac{K_{m g} \cdot K_{s} \cdot\left(I-O_{t}\right)}{1+\left|K_{m g} K_{g m}\right| \cdot K_{s}^{2}}
\end{aligned}\right.
$$

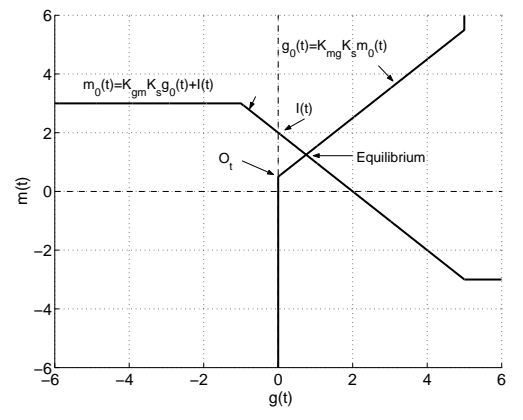

Fig. 5. The nullclines.

\section{B. Case II:}

If we want to keep the original position of the nonlinear function. The simplest model resulting in the desired system response is depicted in Fig. 6. A second line is added around

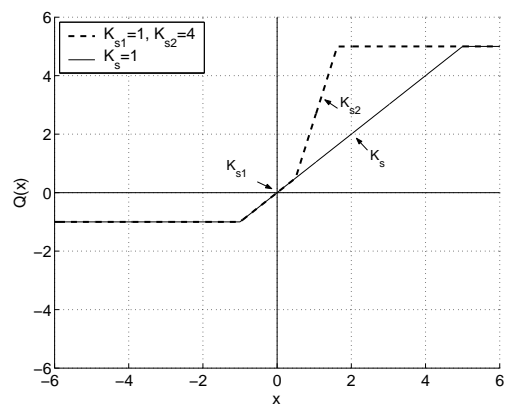

Fig. 6. Nonlinear function. zero that has a smaller slope than the original line. Then the modified nonlinearity is given by

$$
Q(x)= \begin{cases}-1 & x \leq x_{1} \\ K_{s 1} \cdot x & x_{1}<x \leq x_{2} \\ K_{s 2} \cdot x & x_{2}<x \leq x_{3} \\ Q_{m} & x \leq x_{3}\end{cases}
$$

The modified nonlinearity is shown with $\left(x_{1}=-1, x_{2}=\right.$ $\left.0.5, x_{3}=1.625, K_{s 1}=1, K_{s 2}=4\right)$. In order to satisfy the peculiar behavior of the reduced KII set, it is required to choose $K_{m g}$ and $K_{g m}$ satisfying

$$
\left|K_{m g} \cdot K_{g m}\right|<\frac{1}{K_{s 1}^{2}} \cdot \frac{(a+b)^{2}}{a \cdot b} \text { (Any equilibrium around }
$$
zero is stable)

$$
\left|K_{m g} \cdot K_{g m}\right|>\frac{1}{K_{s 2}^{2}} \cdot \frac{(a+b)^{2}}{a \cdot b} \text { (Any other equilibrium is }
$$
unstable)

\section{Computer Simulations}

In this section, we will provide computer simulations on the modified reduced KII set to justify our mathematical results. We implemented the reduced KII set with modified nonlinearity using the digital simulator whose validity we justified previously [7].

For the first set of simulations, we use the nonlinearity given by Eq. (5) with the following set of parameters. $a=220, b=$ $720 ; x_{1}=-0.25 ; x_{2}=1.25 ; K_{s}=4 ; K_{m g}=1 ; K_{g m}=-1$. For the first 0.3 seconds, the input, I, is 3 , and for the rest of the simulation, it is dropped to 0. First, we have to check Eq. (7) to find the range of input values for which the equilibrium is in the linear region of the nonlinearity. Using Eq. (7), we get the range of the input for linear operation $-1.0625<I<5.3125$. Therefore, for the input values that we use, the equilibrium is in the linear region of the nonlinear function. Now, the stability of both points is determined by Eq. (11)

$$
1=\left|K_{m g} \cdot K_{g m}\right|>\frac{1}{K_{s}^{2}} \cdot \frac{(a+b)^{2}}{a \cdot b}=0.3486
$$

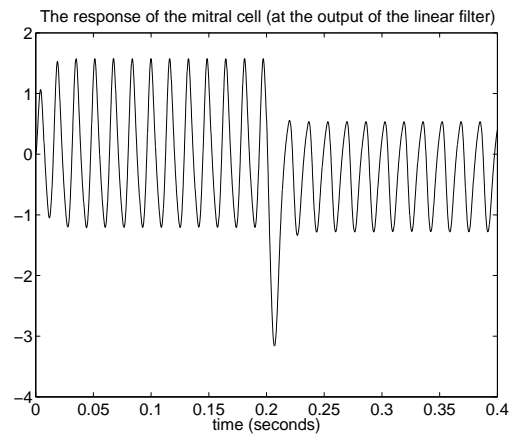

Fig. 7. Time development of mitral cell. $Q(x)$ is described by Eq. (5).

Hence, the solution is unstable. We expect to have oscillations with this given set of parameters for both input values. Fig. 7 shows the response of the mitral cell at the 


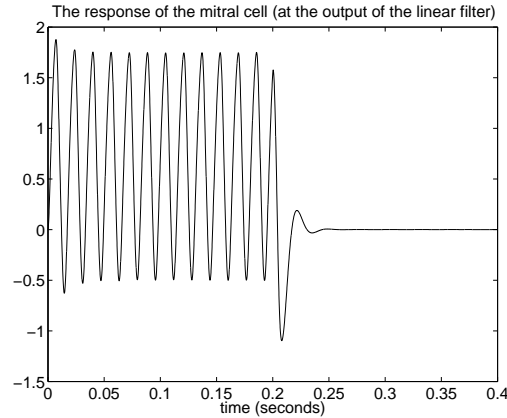

Fig. 8. Time development of mitral cell. $Q(x)$ is described by Eq. (15).

output of the linear filter for the given set of parameters. As observed from this plot, the system exhibits limit cycle behavior for both inputs as we expected. This is mainly because the nonlinear function has the same slope for both equilibrium points. However, in Freeman model, we require the system to converge to stable equilibrium for zero input.

In the second set of simulation, we aim to overcome the stated problem. In accordance with the analysis in section III, we will use the nonlinearity depicted in Fig. 6. Again, we will use the same set of parameters and input signal. In Fig. 6, we have chosen the slope of line around zero to be 1 , and the region of support of that line is between -1 and 1 . We require that the slope of this line be smaller than the original line, which has the slope 4 . The region of support of the added linearity on the $\mathrm{x}$-axis defines the noise margin of the system because the longer the region of support, the higher the input value that causes oscillation. Mathematically,

$$
\left|K_{m g} \cdot K_{g m}\right|<\frac{1}{K_{s 1}^{2}} \cdot \frac{(a+b)^{2}}{a \cdot b}=\frac{(a+b)^{2}}{a b} \frac{1}{1^{2}}=5.5783
$$

$$
\text { (any equilibrium around zero is stable ) }
$$

$$
\left|K_{m g} \cdot K_{g m}\right|>\frac{1}{K_{s 2}^{2}} \cdot \frac{(a+b)^{2}}{a \cdot b}=\frac{(a+b)^{2}}{a b} \frac{1}{4^{2}}=0.3486
$$

(any other equilibrium is unstable )

The response of the mitral cell at the output of the linear filter for the given set of parameters is shown in Fig. 8 . As observed from the figure, with the nonzero input, the system exhibits limit cycle behavior, whereas the output also vanishes when the input is decreased back to zero. This result agrees with our mathematical analysis and matches with the peculiar behavior of the original reduced KII set. That is, the response of the reduced KII set to an impulse input is a damped oscillation whereas with a sustained input, the output oscillation is maintained as long as the input remains.

\section{Modified Freeman Model as an Associative MEMORY}

We have developed the mathematical background for the modified Freeman Model. In this section, we will show the power of the system with an application. Previously, we have shown in [9] that the reduced KII network of the Freeman Model can be used as an auto-associative memory. Here, we will show that the reduced KII network with the modified nonlinear function can still function as an auto-associative memory. The advantage of the new model is the availability of full stability analysis allowing the designer to choose the system parameters properly.

The goal of an auto-associative memory is to store a set of patterns during learning and retrieve the stored pattern that is most similar to the present input pattern. In the literature, there are different feed-forward and recurrent structures that implement associative memory. The famous Hopfield network [12] is a recurrent associative memory having stable point attractors. On the other hand, in Freeman model, the system has non-convergent dynamics with two separate modes of operation. These modes are the stable fixed point and the limit cycle operations, which enables the network to encode information into different dynamical states.

We consider binary input patterns that are multidimensional and static. In other words, these patterns do not change with time and can take only two values. We use the reduced KII network with modified nonlinearity as the auto-associative memory. Each bit in the pattern is applied as the input to one of the reduced KII set. Hence, the dimensionality of the input is equal to the number of reduced KII sets in the reduced KII network. For example, to store the following two patters, 001111 and 111100 , we need to have a network of 6 reduced KII sets. According to the Freeman model, the inhibitory connection weights between reduced KII sets are fixed whereas the excitatory connection weights are trainable. These adaptive excitatory connections are trained with Oja's learning rule [9], which is a stable version of the Hebbian learning. In Oja's rule, we normalize the weights such that the sum of weights connected to a particular input stays constant during training. The normalization constant, which is the sum of the weights connected to each input, has to be selected prior to training.

If a group KII sets are connected by strong excitatory interconnections, then this group will tend to oscillate together: when one KII is applied a strong external input, it induces a strong response in the other nodes that are connected to it. Thus, even if only one member of a set is given a strong input the entire group will oscillate together and in synchrony. If, on the other hand, two nodes are connected by very weak excitatory connections then an oscillation in one set will inhibit oscillations in the other set because the inhibitory interconnections will dominate. Since Freeman model is a dynamical system, the signals at the nodes of the model will not be static. In order to have a static read-out, we can process the signals generated by the mitral cells of the reduced KII network. We perform series of operation which extracts the energy of the dynamical signal. For details of associative memory operation, please refer to [9].

The KII network that we used in the network has the nonlinearity given by Fig. 6

$$
Q(x)= \begin{cases}-1 & x \leq-1 \\ x & -1<x \leq 0.1 \\ 4 \cdot x & 0.1<x \leq 1.325 \\ 5 & x \leq 1.325\end{cases}
$$




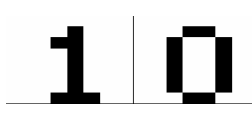

Fig. 9. The stored patterns.

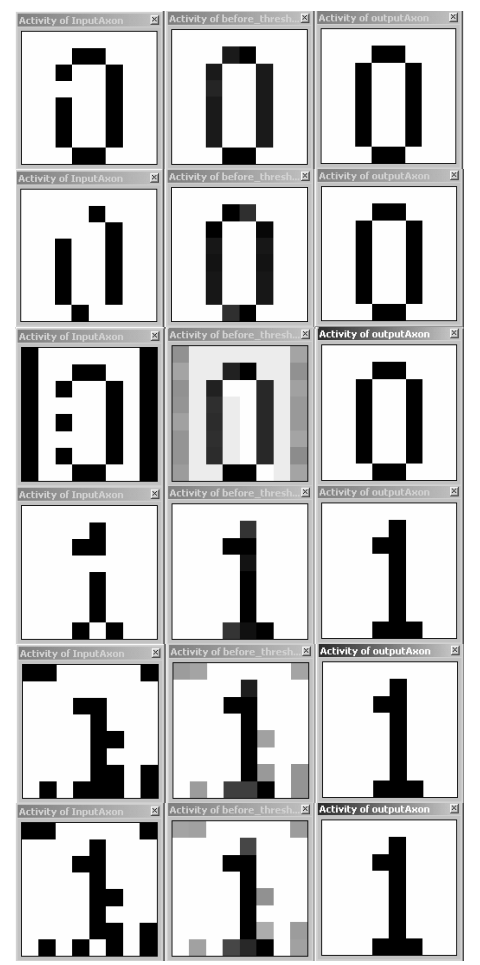

Fig. 10. Recall of the corrupted patterns: The first column is the input to the KII network, the second column is the output before the threshold detector, and the third column is the output after the threshold detector.

We used 64 reduced KII sets, which are fully connected by both excitatory and inhibitory connections. The binary encoded patterns are presented as the inputs to the input terminals of the 64 mitral cells. The two values of the binary input, logic zero and logic one, are encoded with the input levels 0 and 0.3 , respectively. We stored the two patterns shown in Fig. 9. Each pattern is defined by an $8 \times 8$ matrix where each binary entry of the matrix defines the color of the pixel, white corresponding to a logic zero and black corresponding to a logic one. While presenting the patterns during training, the excitatory connection weights are trained using Oja's rule. The internal connection weights and the inhibitory connection weights are always kept constant. The internal connection weights, $K_{m g}$ and $K_{g m}$, are chosen to be 1 and -1 , all the inhibitory connection weights are chosen experimentally at -0.06 [1], [9]. The normalization value for Oja's learning rule is set to be 0.5. After training, all the weight values are fixed and the system is tested with corrupted patterns that have missing pixels in the pattern and extra pixels in the background. In Fig. 10 we show the system response for various corrupted input patterns. As we see in Fig. 10, the KII network with modified nonlinearity can recover the associated stored pattern.

\section{Conclusions}

The general trend in neural engineering is to incorporate biologically realistic neural network models to the design of engineering systems. Freeman Model, a dynamical model of the olfactory cortex, an area responsible for processing sensory information, is one of these biological models. Previously, it has been shown that the Freeman Model can be used as an associative memory. However, due to the nature of the nonlinear function in the model, understanding the behavior of this complex dynamical system is mainly limited to computer simulations. In this paper, we propose to replace the nonlinear function with a piecewise linear approximation. We show that this modified system still preserves the intrinsic behavior of the original Freeman model. With this modified nonlinearity, the control parameters of the basic neural oscillator can be determined analytically. Moreover, we used this modified model as an auto-associative memory which can store a set of patterns during learning and retrieve the stored pattern that is most similar to the present input pattern. Future work will expand the mathematical results obtained to more complicated KII network. We will also compare the capacity and accuracy of recall of the modified system used as an associative memory with the original system.

\section{ACKNOWLEDGMENT}

This work was partially supported by ONR N00014-1-10405 .

\section{REFERENCES}

[1] Y. Yao, W. Freeman, B. Burke, and Q. Yang, "Pattern recognition by a distributed neural network: An industrial application," International Journal of Bifurcation and Chaos, vol. 11, no. 6, pp. 1607-1629, 1991.

[2] R. Kozma and W. Freeman, "Chaotic resonance-methods and applications for robust classification of noisy and variable patterns," Neural Networks, vol. 4, no. 1, pp. 103-121, 2001.

[3] L. Chua and L. Yang, "Cellular neural networks: Theory and applications," IEEE Trans. Circuits Syst., vol. 35, no. 10, pp. 1257-1290, Oct. 1988.

[4] D. Wang, "Relaxation oscillators and networks," in Wiley Encyclopedia of Electrical and Electronics Engineering, J. G. Webster, Ed. New York: Wiley Sons, 1999, pp. 396-405.

[5] R. D. Henkel, "Segmentation with synchronizing neural oscillators," Center for Cognitive Science, Bremen, Tech. Rep. ZKW-Report, Apr. 1994.

[6] W. Freeman, Mass Action in the Nervous System. New York: Academic, 1975.

[7] M. Ozturk, J. Principe, B. Davis, and D. Erdogmus, "Simulation of the freeman model of the olfactory cortex: A quantitative performance analysis for the dsp approach," in Proc. International Joint Conference on Neural Networks (IJCNN'03), vol. 1, Portland, USA, July 2003, pp. $332-336$.

[8] J. Principe, V. Tavares, J. Harris, and W. Freeman, "Design and implementation of a biologically realistic olfactory cortex in analog VLSI," Proc. IEEE, vol. 89, no. 7, pp. 569-571, July 2001.

[9] D. Xu, B. Davis, M. Ozturk, L. Deng, M. Skowronski, J. G. Harris, W. J. Freemand, and J. Principe, "Computational neural networks," in Neural Engineering, B. He, Ed. New York: Kluwer (in press).

[10] D. Xu and J. Principe, "Dynamical analysis of neural oscillators in an olfactory cortex model," accepted by IEEE Trans. Neural Networks.

[11] Y. Kuznetsov, L. Kuznetsov, and J. Marsden, Elements of Applied Bifurcation Theory, 2nd ed. New York: Springer-Verlag, 1998.

[12] J. Hopfield, "Neurons with graded response have collective computational properties like those of two-state neurons," Proceedings of the National Academy of Sciences, vol. 81, pp. 3088-3092, 1984. 